\title{
Multiagent PET for Risk Characterization in Sarcoma
}

\author{
Janet F. Eary ${ }^{1,2}$, Jeanne M. Link ${ }^{1}$, Mark Muzi ${ }^{1}$, Ernest U. Conrad ${ }^{2}$, David A. Mankoff ${ }^{1}$, Jedediah K. White ${ }^{2}$, \\ and Kenneth A. Krohn ${ }^{1}$ \\ ${ }^{1}$ Department of Radiology, University of Washington, Seattle, Washington; and ${ }^{2}$ Department of Orthopedics and Sports Medicine, \\ University of Washington, Seattle, Washington
}

\begin{abstract}
A major goal of molecular imaging in cancer is to evaluate patient tumors for risk of treatment resistance and poor outcome using biologically specific PET agents. This approach was investigated using a multiagent imaging protocol for which patients were imaged in a single session to minimize changes in tumor parameters caused by multiple-day and -setting observation differences. Methods: We present data from a pilot study in 10 soft-tissue sarcoma patients imaged with ${ }^{11} \mathrm{C}$-thymidine for cellular proliferation, ${ }^{18} \mathrm{~F}$-fluoromisonidazole (FMISO) for tissue hypoxia, and ${ }^{11} \mathrm{C}$-verapamil for $\mathrm{P}$-glycoprotein activity, in comparison with ${ }^{15} \mathrm{O}$-water for blood flow and ${ }^{11} \mathrm{C}-\mathrm{CO}_{2}$ for metabolite analysis and ${ }^{18} \mathrm{~F}-\mathrm{FDG}$ clinical scans. Several patients underwent repeated imaging after adriamycin-based chemotherapy. Results: Quantitative imaging results showed that tumor uptake parameters vary between patients and with respect to each other in individual patients, suggesting that each patient's tumor biologic profile is unique. Specific tumor characteristics such as variable cellular proliferation, hypoxic volume, and upregulated P-glycoprotein activity were identified. Conclusion: This study shows that multiagent PET is feasible and yields unique and potentially complementary biologic information on individual tumors.
\end{abstract}

Key Words: PET; ${ }^{11} \mathrm{C}$-verapamil; FMISO; ${ }^{11} \mathrm{C}$-thymidine; sarcoma

J Nucl Med 2011; 52:541-546

DOI: 10.2967/jnumed.110.083717

$\mathbf{T}$ he pathobiologic process of cancer is dynamic and multifaceted. Contributions from clinical and biomedical research continue to add dimensions to this understanding. In clinical practice, risk assessment for cancer outcome is required for patient counseling and treatment planning. Currently, we assess risk with standard clinical methods including physical examination, clinical evaluation, imaging, and tumor biopsy. These evaluations provide information on cancer biologic process parameters but are limited by the discontinuous nature of the observations. These fragmented data likely lack specificity to suggest the most ef-

\footnotetext{
Received Sep. 29, 2010; revision accepted Jan. 7, 2011.

For correspondence or reprints contact: Janet F. Eary, University of Washington Medical Center, 1959 NE Pacific St., Rm. AA011, P.O. Box 356113, Seattle, WA 98195-6113.

E-mail: jeary@u.washington.edu

COPYRIGHT @ 2011 by the Society of Nuclear Medicine, Inc.
}

fective therapy for a patient's treatment. Because of the ability of PET methods to noninvasively report on the rate of change of biologically specific processes, we designed an experimental protocol using sequentially administered ${ }^{11} \mathrm{C}$ and ${ }^{18} \mathrm{~F}$ imaging agents to gauge multiple parameters for patient risk assessment in a single imaging session. The short half-life of ${ }^{11} \mathrm{C}$ is advantageous for sequential radiotracer injection and imaging, as demonstrated by our previous experience $(1-5)$.

The soft-tissue sarcoma patient population under care in our institution comprises a group with highly diverse tumor biology. Despite this diversity, treatment protocols are the same for most soft-tissue sarcoma types. This fact points out the need for identification of tumors at high risk for poor outcome within this group. Sarcomas exhibit intralesional heterogeneity in cellular proliferation rates, making it difficult to predict treatment effectiveness. These differences led us to choose this tumor group to demonstrate that diverse tumors will show differences in specific imaging agent uptake parameters. For example, a high level of cellular proliferation is a feature of high-grade soft-tissue sarcomas, suggesting that treatment with cell cycle-active cytotoxic chemotherapy agents would be effective. Lowergrade tumors are less likely to have high cellular proliferation rates and are known to be less responsive to standard chemotherapy. Measurement of this parameter before and during therapy would be useful in planning individualized treatment and monitoring early response. Sarcomas also have intrinsic multiple-drug resistance, a significant problem for effectiveness of alkylator drugs, which form the basis of sarcoma chemotherapy. This resistance is thought to be induced as a consequence of therapy. Many sarcomas are also known to be hypoxic, which likely confers resistance to chemo- and radiotherapy and accelerated metastatic spread. Many patients experience tumor local recurrences, but pulmonary metastases are the principal cause of death. Consequently, neoadjuvant and adjuvant treatments in sarcoma are aimed primarily at decreasing pulmonary metastases or treating those metastases that present early in the patient's disease course. Tumor biologic features may also directly influence the risk for pulmonary metastases and their progression. The multiagent PET protocol we developed was designed to demonstrate the use of multiple biologic measures for risk associated with tumor biologic 
TABLE 1

PET Agents for Risk Stratification in Sarcoma

\begin{tabular}{|c|c|}
\hline $\begin{array}{l}\text { Imaging } \\
\text { agent }\end{array}$ & $\begin{array}{c}\text { Biologic } \\
\text { assessment }\end{array}$ \\
\hline${ }^{11} \mathrm{C}$-thymidine & $\begin{array}{l}\text { Tumor } \\
\text { proliferation }\end{array}$ \\
\hline${ }^{18} \mathrm{~F}$-fluoromisonidazole & $\begin{array}{l}\text { Tissue } \\
\text { hypoxia }\end{array}$ \\
\hline${ }^{11} \mathrm{C}$-verapamil & $\begin{array}{l}\text { Tissue } \mathrm{P} \text {-gp } \\
\text { (multidrug resistance) }\end{array}$ \\
\hline${ }^{15} \mathrm{O}$-water & $\begin{array}{l}\text { Regional } \\
\text { blood flow }\end{array}$ \\
\hline${ }^{11} \mathrm{C}-\mathrm{CO}_{2}$ & $\begin{array}{l}\text { Tissue } \mathrm{pH} \text {, } \\
\text { thymidine metabolite } \\
\text { correction }\end{array}$ \\
\hline
\end{tabular}

characteristics. This PET tumor imaging methodology may be an effective tool for risk assessment in individual patients with many other types of cancer.

Three PET agents provided the core of the imaging sequence in this study: ${ }^{11} \mathrm{C}$-thymidine, for tissue cellular proliferation; ${ }^{11} \mathrm{C}$-verapamil, a P-glycoprotein (P-gp) substrate, to assess multiple-drug resistance activity; and ${ }^{18} \mathrm{~F}-$ fluoromisonidazole $\left({ }^{18} \mathrm{~F}\right.$-FMISO), to identify tumor hypoxic volume (Table 1). This group of imaging agents was supported by additional imaging with ${ }^{15} \mathrm{O}$-water, an indicator of tumor blood flow, and ${ }^{11} \mathrm{C}_{-} \mathrm{CO}_{2}$, the dominant labeled metabolite of thymidine. In this report, initial experience in the feasibility of multiagent PET studies for assessment of the dynamic cancer biologic process is presented.

\section{MATERIALS AND METHODS}

\section{Patients}

Adult patients with soft-tissue sarcoma from our clinical service who were planning to undergo neoadjuvant chemotherapy were invited to participate in the study. This study was performed under an approved institutional protocol for which patients provided written informed consent to participate. The patients and their diagnoses, with baseline tumor dimensions, are listed in Table 2. They were imaged before the start of neoadjuvant adriamycin-based chemotherapy, after the first therapy cycle, and after the completion of therapy, in the week before resection. ${ }^{11} \mathrm{C}$-verapamil imaging was omitted in the mid-therapy imaging session. Figure 1 shows the imaging protocols in the therapy sequence.

\section{Radiopharmaceuticals}

${ }^{11} \mathrm{C}$-thymidine, ${ }^{11} \mathrm{C}$-verapamil, ${ }^{11} \mathrm{C}-\mathrm{CO}_{2},{ }^{15} \mathrm{O}$-water, and ${ }^{18} \mathrm{~F}$ FMISO were prepared according to previously established methods (6-8). Radiotracers were infused in sequence of isotope decay rate, beginning with ${ }^{15} \mathrm{O}$-water and ending with ${ }^{18} \mathrm{~F}$-FMISO. The total radiation dose exposure for 1 full imaging session with this protocol, including an attenuation scan, was $1.0 \mathrm{rad}$, compatible with the guidelines of the Food and Drug Administration Radioactive Drug Research Committee.

\section{PET}

Imaging occurred in 2- to 4-h sessions for each patient. This protocol was designed to simplify image registration because the patient did not move between radiopharmaceutical injections. Each imaging sequence was performed in a single imaging session. The PET agents were infused and imaged sequentially while the patient rested comfortably on the imaging table. The exception was for the delayed ${ }^{18} \mathrm{~F}$-FMISO images for which the patient was repositioned for a second image after a 90-min rest period. A urinary bladder catheter was placed for patients with tumors in the pelvis and proximal thigh. An intravenous line for injection of the radiotracer was placed if an intravenous infusion port was not available. An additional intravenous or arterial line was placed for sequential blood sampling during the imaging sequences. Imaging was performed with dynamic acquisition according to the imaging times shown in Table 3 . The imaging acquisition times were determined by the biodistribution and biologic behavior of each imaging agent and are the minimum time for acquisition of reliable dynamic data for assessment of tissue uptake rates.

The Advance PET (GE Healthcare) scanner operating in 2-dimensional high-sensitivity mode with 35 image planes (plane thickness, $4.25 \mathrm{~mm}$ ) per axial field of view of $15 \mathrm{~cm}$ was used to acquire the images. Our Advance scanner has been refurbished so that the PET data acquisition and processing are the same as those used in current PET/CT units, such as the Discovery LS (GE

TABLE 2

Patient Characteristics

\begin{tabular}{|c|c|c|c|c|}
\hline Patient no. & Diagnosis & Location & Grade & Size $^{\star}(\mathrm{cm})$ \\
\hline 1 & Undetermined $^{\dagger}$ & Right flank & & $13.3 \times 7.6 \times 15.2$ \\
\hline 2 & Liposarcoma & Thigh & 3 & $8.0 \times 7.0 \times 5.0$ \\
\hline 3 & Myofibrosarcoma & Forearm & 2 & $3.8 \times 2.7 \times 5.5$ \\
\hline 4 & Myofibroblastic sarcoma & Pelvis & 3 & $5.5 \times 6.7 \times 14.9$ \\
\hline 5 & Alveolar rhabdomyosarcoma & Forearm & 3 & $15.0 \times 6.0 \times 5.0$ \\
\hline 6 & Synovial sarcoma & Thigh & 3 & $8.0 \times 6.9 \times 11.1$ \\
\hline 7 & Liposarcoma & Pelvis & 2 & $13.0 \times 10.0 \times 10.0$ \\
\hline 8 & Leiomyosarcoma & Gluteus & 3 & $9.5 \times 6.6 \times 6.1$ \\
\hline 9 & Myxofibrosarcoma & Gluteus & $2-3$ & $3.1 \times 6.0 \times 5.6$ \\
\hline 10 & Pleomorphic sarcoma & Thigh & 3 & $5.7 \times 6 \times 5.6$ \\
\hline
\end{tabular}

*Tumor size in 3 dimensions.

†Initially diagnosed as sarcoma, later, unclear tumor type.

${ }^{\ddagger}$ Alveolar rhabdomyosarcoma is a high-grade tumor but not usually assigned an FNLCC tumor grade. 


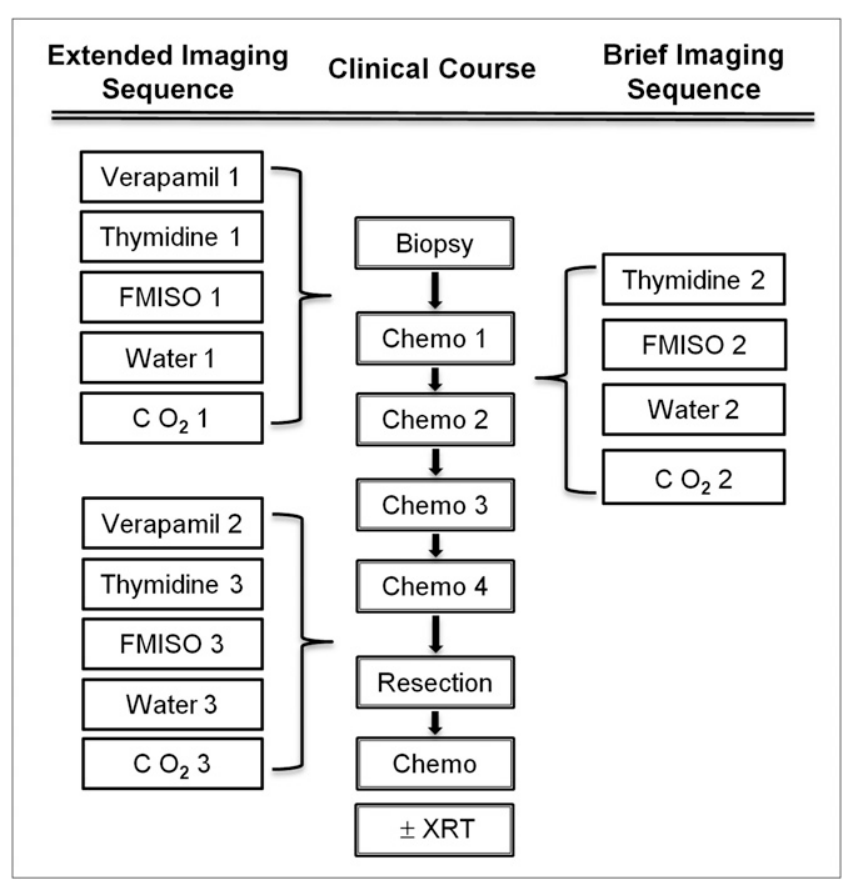

FIGURE 1. Multiagent imaging sequence. XRT = radiation therapy.

Healthcare). The main difference is that a 25-min attenuation scan is required over the body region of interest, and this was also acquired during the imaging sequence. This attenuation procedure involved a radiation exposure of $25 \mathrm{mrem}$. The use of the dedicated PET scanner (Advance) had the advantage of reducing radiation-absorbed dose to the patient, in keeping with the principle of dose reduction whenever possible while maintaining imaging data quality.

Sequential blood samples acquired during each imaging session were centrifuged, and plasma aliquots were counted in a well counter (Cobra II; Packard Instrument Co.). ${ }^{11} \mathrm{C}$-thymidine blood samples were additionally analyzed by acid precipitation and high-performance liquid chromatography for determination of the fractions of labeled metabolites using previously published methods (9). ${ }^{11} \mathrm{C}-\mathrm{CO}_{2}$ samples were analyzed by acid precipitation in the same manner as for thymidine.

\section{Image Data Analysis}

Emission images were corrected for random and scattered coincidence events. Each dynamic image acquisition dataset was decay-corrected for the radiolabel. If necessary, datasets were also corrected for residual activity of the previously imaged radiotracer, as measured in the last frame of the preceding scan.

Volumes of interest hand-drawn by an operator were used to analyze PET data. Volume-of-interest placement for tumor regions was determined on each summed image set for each radiotracer acquisition by including voxels with values greater than half of the maximum tumor value, corrected for background. These regions were identified within the tumor images themselves and compared visually to the corresponding ${ }^{18} \mathrm{~F}$-FDG images. Repeated images in each patient were analyzed in a similar manner. Tissue timeactivity curves from dynamic image data were generated for these regions of interest. Arterial blood samples were taken when possible. Samples taken intravenously were used to rescale a historic arterial input function assuming arteriovenous equilibration at later times. Historic data for metabolite correction for ${ }^{11} \mathrm{C}$-thymidine and ${ }^{11} \mathrm{C}$-verapamil were also available in those instances for which metabolite analysis failed because of procedural error or failure of the blood sampling line. Blood sample data were corrected for residual activity of the previously imaged radiotracer in the instances that required such a correction.

The previously published 5-tissue compartmental model for determination of thymidine incorporation rate in somatic tissues was used for this analysis $(3,10)$. Rate parameters for metabolites were set to the values found from analysis of ${ }^{11} \mathrm{C}-\mathrm{CO}_{2}$ uptake rates in a separate 2-tissue compartmental model (10).

${ }^{15} \mathrm{O}$-water provided blood flow $(\mathrm{mL} / \mathrm{g} / \mathrm{min})$ information for comparison with ${ }^{11} \mathrm{C}$-verapamil transport and was modeled using a 1-tissue compartmental model for blood flow with dispersion corrections (11). ${ }^{11} \mathrm{C}$-verapamil tumor uptake was assessed by a simple 1-tissue compartmental model using the early part of the uptake data $(10 \mathrm{~min})$ to determine the initial transport $\left(\mathrm{K}_{1} ; \mathrm{mL} / \mathrm{g} / \mathrm{min}\right)$ and distribution $\left(\mathrm{Vd}=K_{1} / k_{2}\right)(12)$. Because the initial transfer of verapamil into tissue is influenced by blood flow, verapamil transport was normalized by blood flow ( $K_{1}$ /flow, unitless) determined from ${ }^{15} \mathrm{O}$-water. ${ }^{18} \mathrm{~F}$-FMISO imaging information was analyzed for determination of tumor hypoxic volume in cubic centimeters using a previously published method (13). This analysis requires a static image and venous blood sampling at the time of the delayed image acquisition.

\section{RESULTS}

Ten patients participated in this feasibility study. Five patients $(2,5,6,7$, and 8$)$ completed the entire imaging protocol. Patient 1 was imaged only at baseline because, after extensive pathologic review, her tumor histologic type

TABLE 3

Imaging Study Sequence

\begin{tabular}{lcl}
\hline \multicolumn{1}{c}{ Procedure } & Time $(\mathrm{min})$ & \multicolumn{1}{c}{ Dose } \\
\hline Patient positioning (1 field of view) & 3 & \\
Transmission imaging & $20-25$ & \\
${ }^{15} \mathrm{O}-$ water & 10 & $25.9 \mathrm{MBq} / \mathrm{kg}(0.7 \mathrm{mCi} / \mathrm{kg})(1,850 \mathrm{MBq}[50 \mathrm{mCi}] \mathrm{maximum})$ \\
${ }^{11} \mathrm{C}-\mathrm{CO}_{2}$ & 60 & $7.4 \mathrm{MBq} / \mathrm{kg}(0.2 \mathrm{mCi} / \mathrm{kg})(444 \mathrm{MBq}[12 \mathrm{mCi} \mathrm{maximum})$ \\
${ }^{11} \mathrm{C}-$ thymidine & 60 & $7.4 \mathrm{MBq} / \mathrm{kg}(0.2 \mathrm{mCi} / \mathrm{kg})(518 \mathrm{MBq}[14 \mathrm{mCi} \mathrm{maximum})$ \\
${ }^{11} \mathrm{C}$-verapamil & 45 & $7.4 \mathrm{MBq} / \mathrm{kg}(0.2 \mathrm{mCi} / \mathrm{kg})(740 \mathrm{MBq}[20 \mathrm{mCi}] \mathrm{maximum})$ \\
${ }^{18} \mathrm{~F}-$ FMISO & (at $90 \mathrm{~min}) 20 \mathrm{~min}$ & $259 \mathrm{MBq}(7 \mathrm{mCi})$ \\
\hline
\end{tabular}




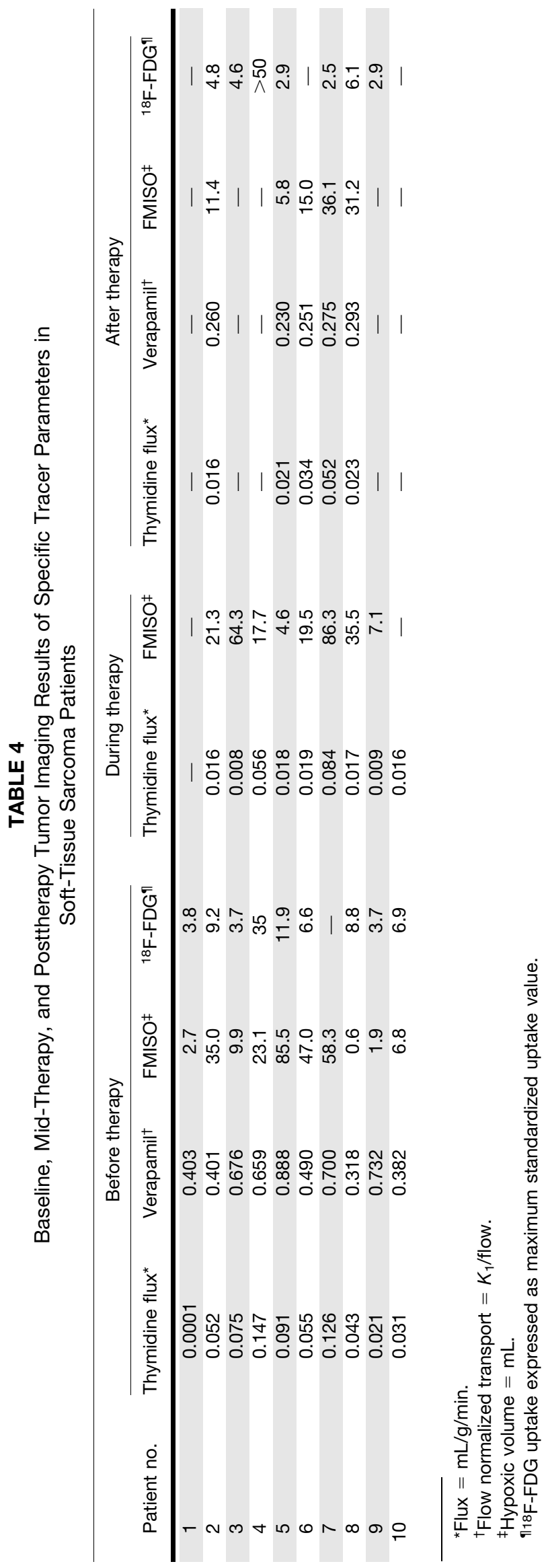

was changed to uncertain for sarcoma. The remaining 4 patients $(3,4,9$, and 10) did not participate in the third imaging session for reasons including chemotherapy toxicity or patient unavailability for the scan. One of these patients (10) did not undergo the second imaging session because of toxicity from chemotherapy. Two patients ( 2 and 8 ) were not able to return after 1 cycle, because they lived over 400 miles from the imaging center, but were imaged after 2 cycles of chemotherapy and before tumor resection. One patient (7) had already received a maximum dosage of adriamycin-based therapy in prior treatment and was given high doses of ifosfamide.

The multiparameter tumor uptake data are shown in Table 4 . The pretreatment tumor values were variable, with coefficients of variation (COVs) of $86 \%$ for thymidine flux, $81 \%$ for ${ }^{18} \mathrm{~F}$-FMISO hypoxic volume, and $34 \%$ for P-gp volume. This finding reflects the biologic variability of sarcomas, which probably affects patient outcome. There was no association between the patient tumor grades and the imaging parameters and little association between the parameters themselves.

The range of $K_{1} /$ flow values for the tumor region of interest in pretreatment sarcoma studies ranged from 0.318 to 0.888 (mean, 0.565 ; COV, $32 \% ; n=10$ ), whereas the posttreatment studies had a considerably lower range, from 0.230 to 0.293 (mean, 0.262; COV, 8\%; $n=5$ ). For the 5 patients with completed studies, the initial transport of ${ }^{11} \mathrm{C}$-verapamil $\left(K_{1}\right)$, which is associated with $\mathrm{P}$-gp activity, ranged from 0.074 to $0.293 \mathrm{~mL} / \mathrm{g} / \mathrm{min}$ (mean, $0.149 \mathrm{~mL} / \mathrm{g} / \mathrm{min}$; COV, $53 \%$ ) for the studies before therapy and from 0.038 to $0.093 \mathrm{~mL} / \mathrm{g} / \mathrm{min}$ (mean, $0.069 \mathrm{~mL} / \mathrm{g} / \mathrm{min}$; COV, 30\%) for the studies after therapy. The average decline (43\%) between pre- and posttherapy verapamil $K_{1}$ for these 5 patients was similar to the average decline $(45 \%)$ in $K_{1}$ /flow, indicating that blood flow was relatively stable between studies.

Eight patients underwent follow-up imaging after their baseline pretreatment scans. These data, shown in Table 5, did not identify a clear association of changes between baseline tumor and follow-up imaging values for any of the parameters. Most patients showed decreases in thymidine flux, indicating response. Several patients also experienced a decline in tumor hypoxic volume, but these changes did not parallel other imaging changes in the patient group. An imaging example from the combined imaging study, displaying variability in tumor uptake and spatial distribution for each of the tracers, is shown in Figure 2.

\section{DISCUSSION}

In this small pilot patient group, none of the imaging parameters showed an association with initial tumor grade, which is the classic histopathologic assessment for risk of metastases and poor outcome. This finding is consistent with our clinical experience and that of other sarcoma clinical groups, supporting the notion that tumor biologic behavior in a patient at presentation is rather more unique 
TABLE 5

Change in Metabolic Parameters

\begin{tabular}{|c|c|c|c|c|c|c|c|c|c|c|}
\hline \multirow[b]{2}{*}{ Metabolic parameter } & \multicolumn{10}{|c|}{ Patient } \\
\hline & 1 & 2 & 3 & 4 & 5 & 6 & 7 & 8 & 9 & 10 \\
\hline \multicolumn{11}{|l|}{ Baseline to during therapy } \\
\hline$\Delta$ Hypoxic volume & - & $-48 \%$ & $455 \%$ & $442 \%$ & $-98 \%$ & $-51 \%$ & $47 \%$ & $53 \%$ & $244 \%$ & - \\
\hline$\Delta$ Thymidine flux & - & $-70 \%$ & $-89 \%$ & $-62 \%$ & $-80 \%$ & $-65 \%$ & $-33 \%$ & $-60 \%$ & $-57 \%$ & $-48 \%$ \\
\hline \multicolumn{11}{|l|}{ Baseline to after therapy } \\
\hline$\Delta$ Thymidine flux & - & $-70 \%$ & - & - & $-77 \%$ & $-38 \%$ & $-59 \%$ & $-47 \%$ & - & - \\
\hline$\Delta$ Verapamil $K_{1}$ & - & $-44 \%$ & - & - & $-78 \%$ & $-16 \%$ & $-68 \%$ & $-7 \%$ & - & - \\
\hline$\Delta$ Verapamil $K_{1} /$ flow & - & $-35 \%$ & - & - & $-74 \%$ & $-49 \%$ & $-61 \%$ & $-8 \%$ & - & - \\
\hline$\Delta$ Hypoxic volume & - & $-67 \%$ & - & - & $-99 \%$ & $-67 \%$ & $-13 \%$ & $34 \%$ & - & - \\
\hline
\end{tabular}

to an individual patient than could be predicted from a large compilation of pooled clinical data summaries, such as the classification description of tumor grade. There was also little association between the individual parameters themselves. The patient tumors in this small study had highly individualized variations in the biologic parameters studied and demonstrate that multiparameter imaging can be used to highlight these.

The tumor flux values calculated using the compartmental model were within previously published ranges for other tumors such as the glioblastoma dataset (13). The sarcoma patients had thymidine flux values that ranged over 100-fold, from 0.0001 to 0.147 . Likewise, the ${ }^{18} \mathrm{~F}$-FMISO tumor hypoxic volumes were similar to those previously identified (0.06-85.5 mL for the current dataset vs. $0-165.8 \mathrm{~mL}, n=19$, for our previously published sarcoma data (14)). ${ }^{11} \mathrm{C}$-verapamil data acquired in this study are new and present a beginning dataset for survey of P-gp expression among patients with sarcoma and other cancer histologies. Our prior experience examining P-gp activity in the normal brain produced flow-normalized verapamil transport values ( $K_{1} /$ flow) considerably lower than sarcoma-a result that is most likely due to higher blood flow in the normal brain (12).

These data also show that most patients in the group experienced decreases in tumor proliferation and hypoxic volume during neoadjuvant treatment. Tumor proliferation decreased throughout therapy in some patients, whereas this decrease occurred only early in others. This finding is consistent with the clinical view that large sarcomas may require several therapy cycles before a response can be observed. It is also a possibility that soft-tissue sarcoma histologies have different temporal and spatial response patterns. Hypoxia-associated lowered tissue $\mathrm{pH}$ may be a factor that limits the cellular uptake of mildly acidic drugs, such as doxorubicin, in addition to upregulation of tumor stress elements that compose increased malignant behavior.

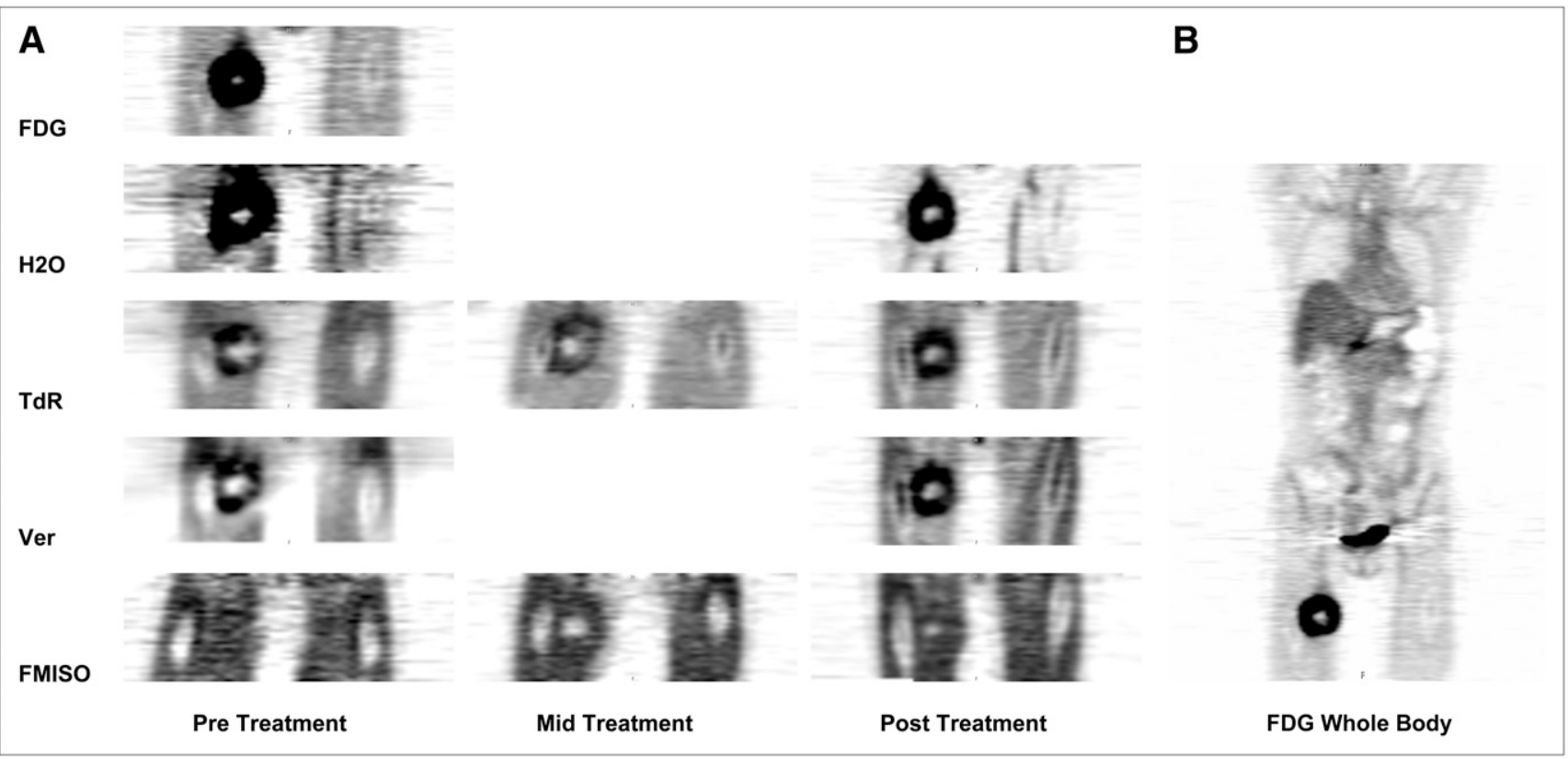

FIGURE 2. Composite images from patient 6 , who completed imaging study. Summed images from single-field-of-view dynamic multiagent imaging studies are shown. These images are scaled to reflect relative uptake values from image data analysis. (A) In these coronal images, tumor is in right proximal thigh. (B) Whole-body ${ }^{18} \mathrm{~F}-\mathrm{FDG}$ image before therapy. $\mathrm{TdR}=$ thymidine; Ver $=$ verapamil. 
Tumor P-gp activity assessed with ${ }^{11} \mathrm{C}$-verapamil analysis methods and results were also compared in this pilot study. The measure of tumor verapamil transport $\left(K_{1} /\right.$ flow $)$ was the parameter chosen for focus in this series. $K_{1} /$ flow is thought to be an accurate reflection of P-gp activity because it accounts for the effects of blood flow on tumor tracer deposition (12).

The group studied is similar to other patient groups who receive toxic neoadjuvant chemotherapy. Several patients studied could not tolerate imaging after completion of the treatment. Similar to other populations referred by academic medical centers, several patients in our study lived some distance away and could not travel because of treatment-related toxicity and travel conditions. This initial experience also suggests that imaging studies relating early baseline tumor characteristics and early response to outcome before treatmentrelated toxicity ensues will enable patient participation. These studies would also include reduced numbers of parameters. In our current protocols, ${ }^{18} \mathrm{~F}$-FMISO and ${ }^{11} \mathrm{C}$-verapamil will be used to focus attention on these tumor parameters that will likely influence treatment decision making.

In this presentation, we demonstrate the feasibility of performing a multiagent PET study for cancer patient risk assessment. Additional feasibility of repeated imaging after treatment initiation and the high level of coordination required between the research group in the radiochemistry area and PET suite is also demonstrated. In short, at this early time point in development of the multiagent PET molecular imaging concept, the complexity and integration of tasks are significant and an important starting point for eventual simplification and refinement of the methods for clinical use. Intriguing imaging findings show that an individual patient tumor phenotype can be observed and quantified repeatedly and pave the way for future larger-scale studies.

\section{CONCLUSION}

Multiagent PET for cancer risk assessment that quantifies tumor proliferation, drug resistance activity, and tissue hypoxia is feasible. The results in soft-tissue sarcoma patients demonstrate that the measured tumor parameters display unique aspects of each individual patient tumor, a finding that may contribute to clinical risk assessment for tumor-aggressive behavior.

\section{DISCLOSURE STATEMENT}

The costs of publication of this article were defrayed in part by the payment of page charges. Therefore, and solely to indicate this fact, this article is hereby marked "advertisement" in accordance with 18 USC section 1734.

\section{ACKNOWLEDGMENT}

This study was supported by NIH P01 CA042045 and NCRR S10 RR17229.

\section{REFERENCES}

1. Mankoff DA, Dunnwald LK, Gralow JR, et al. Changes in blood flow and metabolism in locally advanced breast cancer treated with neoadjuvant chemotherapy. J Nucl Med. 2003;44:1806-1814.

2. Krohn KA, O'Sullivan F, Crowley J, et al. Challenges in clinical studies with multiple imaging probes. Nucl Med Biol. 2007;34:879-885.

3. Wells JM, Mankoff DA, Eary JF, et al. Kinetic analysis of $2-\left[{ }^{11} \mathrm{C}\right]$ thymidine PET imaging studies of malignant brain tumors: preliminary patient results. $\mathrm{Mol}$ Imaging. 2002;1:145-150.

4. Eary JF, Mankoff DA, Spence AM, et al. 2-[C-11]thymidine imaging of malignant brain tumors. Cancer Res. 1999;59:615-621.

5. Spence AM, Muzi M, Graham MM, et al. Glucose metabolism in human malignant gliomas measured quantitatively with PET, 1-[C-11]glucose and FDG: analysis of the FDG lumped constant. J Nucl Med. 1998;39:440-448.

6. Link JM, Bray KE, Krohn KA. Micro-synthesis of 2- $\left({ }^{11} \mathrm{C}\right)$-thymidine to improve yield [abstract]. J Labelled Comp Radiopharm. 2007;50(suppl 1):S207.

7. Elsinga PH, Franssen EJF, Hendrikse NH, et al. Carbon-11-labeled daunorubicin and verapamil for probing P-glycoprotein in tumors with PET. J Nucl Med. 1996;37:1571-1575.

8. Lim J-L, Berridge MS. An efficient radiosynthesis of [F-18]fluoromisonidazole. Appl Radiat Isot. 1993;44:1085-1091.

9. Shields AF, Mankoff D, Graham MM, et al. Analysis of 2-carbon-11-thymidine blood metabolites in PET imaging. J Nucl Med. 1996;37:290-296.

10. Wells JM, Mankoff DA, Muzi M, et al. Kinetic analysis of $2-\left[{ }^{11} \mathrm{C}\right]$ thymidine PET imaging studies of malignant brain tumors: compartmental model investigation and mathematical analysis. Mol Imaging. 2002;1:151-159.

11. Alpert NM, Eriksson L, Chang JY, et al. Strategy for the measurement of regional cerebral blood flow using short-lived tracers and emission tomography. J Cereb Blood Flow Metab. 1984;4:28-34.

12. Muzi M, Mankoff DA, Link JM, et al. Imaging of cyclosporine inhibition of P-glycoprotein activity using ${ }^{11} \mathrm{C}$-verapamil in the brain: studies of healthy humans. J Nucl Med. 2009;50:1267-1275.

13. Krohn KA, Link JM, Mason RP. Molecular imaging of hypoxia. J Nucl Med. 2008;49(suppl 2):129S-148S.

14. Rajendran JG, Wilson DC, Conrad EU, et al. $\left[{ }^{18} \mathrm{~F}\right]$ FMISO and $\left[{ }^{18} \mathrm{~F}\right]$ FDG PET imaging in soft tissue sarcomas:correlation of hypoxia, metabolism and VEGF expression. Eur J Nucl Med. 2003;30:695-704. 